\title{
Time-Dependent Wave Equations on Graded Groups
}

\author{
Michael Ruzhansky ${ }^{1,2}$ (D) Chiara Alba Taranto ${ }^{3}$
}

Received: 3 June 2020 / Accepted: 28 January 2021 / Published online: 4 February 2021

(C) The Author(s) 2021

\begin{abstract}
In this paper we consider the wave equations for hypoelliptic homogeneous leftinvariant operators on graded Lie groups with time-dependent Hölder (or more regular) nonnegative propagation speeds. The examples are the time-dependent wave equation for the sub-Laplacian on the Heisenberg group or on general stratified Lie groups, or $p$-evolution equations for higher order operators on $\mathbb{R}^{n}$ or on groups, already in all these cases our results being new. We establish sharp well-posedness results in the spirit of the classical result by Colombini, De Giorgi and Spagnolo. In particular, we describe an interesting local loss of regularity phenomenon depending on the step of the group (for stratified groups) and on the order of the considered operator.
\end{abstract}

Keywords Sub-Laplacian · Rockland operator · Gevrey spaces · Wave equation · Heisenberg group · Graded groups

Mathematics Subject Classification (2010) $35 \mathrm{~L} 05 \cdot 35 \mathrm{~L} 30 \cdot 43 \mathrm{~A} 70 \cdot 42 \mathrm{~A} 80$

The first author was supported in parts by the EPSRC grants EP/R003025, EP/K039407/1 and by the Leverhulme Grants RPG-2017-151, RPG-2014-02, as well as by the FWO Odysseus 1 grant

G.0H94.18N: Analysis and Partial Differential Equations.

M. Ruzhansky

Michael.Ruzhansky@UGent.be

1 Department of Mathematics: Analysis, Logic and Discrete Mathematics, Ghent University, Ghent, Belgium

2 School of Mathematical Sciences, Queen Mary University of London, London, UK

3 Department of Mathematics, Imperial College London, 180 Queen's Gate, London SW7 2AZ, UK 


\section{Introduction}

In this paper we are interested in the well-posedness of the following Cauchy problem:

$$
\begin{cases}\partial_{t}^{2} u(t, x)+a(t) \mathcal{R} u(t, x)=0, & (t, x) \in[0, T] \times G, \\ u(0, x)=u_{0}(x), & x \in G, \\ \partial_{t} u(0, x)=u_{1}(x), & x \in G,\end{cases}
$$

for the time-dependent propagation speed $a=a(t) \geq 0$, where $\mathcal{R}$ is a Rockland operator (that is, a hypoelliptic homogeneous differential operator on a graded group $G$ ).

In the case of $G=\mathbb{R}^{n}$ and $\mathcal{R}=-\Delta$, Eq. (1.1) is the usual wave equation with the timedependent propagation speed and its well-posedness results for Hölder regular functions $a$ have been obtained by Colombini, De Giorgi and Spagnolo in their seminal paper [12]. Moreover, it has been shown by Colombini and Spagnolo in [11] and by Colombini, Jannelli and Spagnolo in [13] that even in the case of $G=\mathbb{R}$ and $\mathcal{R}=-\frac{d^{2}}{d x^{2}}$ the Cauchy problem (1.1) does not have to be well-posed in $\mathcal{C}^{\infty}$ if $a \in \mathcal{C}^{\infty}$ is not strictly positive or if it in the Hölder class $a \in \mathcal{C}^{\alpha}$ for $0<\alpha<1$.

In this paper we obtain new results for the following situations:

(i) $G=\mathbb{H}^{n}$ is the Heisenberg group and $\mathcal{R}$ is the positive Kohn-Laplacian on $G$.

(ii) $G$ is a stratified Lie group and $\mathcal{R}$ is a (positive) sub-Laplacian on $G$.

(iii) $G$ is a graded Lie group in the sense of Folland and Stein [24] and $\mathcal{R}$ is any positive Rockland operator on $G$, i.e. any positive left-invariant homogeneous hypoelliptic differential operator on $G$.

In fact, our results are for the latter case (iii), the former two cases (i) and (ii) being its special cases. In particular, already in the cases of $G$ being the Euclidean space $\mathbb{R}^{n}$, the Heisenberg group $\mathbb{H}^{n}$, or any stratified Lie group, the case (iii) above allows one to consider $\mathcal{R}$ to be an operator of any order, as long as it is a positive left- (or right-) invariant homogeneous hypoelliptic differential operator. In the case of $\mathbb{R}^{n}$ these cases of so-called $p$-evolution equations have been studied in e.g. [4-6], however, for more restrictive conditions on $a(t)$ than those considered in this paper.

For $a(t) \equiv 1$ and $G$ being the Heisenberg group $\mathbb{H}^{n}$ with $\mathcal{R}$ being the positive subLaplacian, the wave equation (1.1) was studied by Müller and Stein [36] and Nachman [37]. Other noncommutative settings with $a(t) \equiv 1$ have been analysed as well, see e.g. Helgason [30]. For $G$ being a compact Lie group and $-\mathcal{R}$ any Hörmander's sum of squares on $G$ the problem (1.1) was studied in [27] (based on the global analysis on compact Lie groups as developed in [43]), and so the results of the present paper provide a nilpotent counterpart of the results there.

Apart from an independent interest of the subelliptic setting of stratified or graded Lie groups, these settings are the model cases for many corresponding problems for general partial differential operators on manifolds in view of the celebrated lifting theorem of Rothschild and Stein [41].

From the point of view of the time-dependent coefficient $a(t)$, we aim at carrying out the comprehensive analysis, thus distinguishing between the following four cases:

Case 1: $a \in \operatorname{Lip}([0, T])$, with $a(t) \geq a_{0}>0$;

Case 2: $a \in \mathcal{C}^{\alpha}([0, T])$, with $0<\alpha<1, a(t) \geq a_{0}>0$;

Case 3: $a \in \mathcal{C}^{l}([0, T])$, with $l \geq 2, a(t) \geq 0$;

Case 4: $a \in \mathcal{C}^{\alpha}([0, T])$, with $0<\alpha<2, a(t) \geq 0$. 
The first case is the simplest situation while in the forth case we have an irregular coefficient that is allowed to be zero at some points. The second and third situations are 'intermediate' cases, in the sense that we have either the regularity or the strict positivity. We distinguish between these cases because the results and methods of proofs are rather different.

We note that if the operator $\mathcal{R}$ is not elliptic, the local approach to the Cauchy problem (1.1) is problematic since the equation is only weakly hyperbolic already in Case 1 above. Consequently, since Eq. (1.1) in local coordinates is the space-dependent variable multiplicities problem, very little is known about its well-posedness. In this direction, only very special results for some second order operators are available, see e.g. Nishitani [38] or Melrose [34]. Non-Lipschitz coefficients have been also much analysed, see e.g. Colombini and Métivier [9] or Colombini and Lerner [8]. In addition to already mentioned restrictions for the well-posedness, see also Colombini and Métivier [10] for a recent overview from the point of view of systems.

In the case of $\mathbb{R}^{n}$ and $-\mathcal{R}$ being the Laplacian, the regularity of $a$ less than Hölder such as discontinuous or measure-valued $a$ have been considered in [28]. However, such low regularity requires very different methods, and this problem for the general Cauchy problem (1.1) will be considered elsewhere.

Wave equations with time dependent coefficients for general densely defined operators with discrete spectrum acting in Hilbert spaces have been considered in [42]. However, that setting is different from the present one since the spectrum in our situation is continuous.

To formulate our results, let us briefly introduce some necessary notations following, for example, Folland and Stein [24]. Let $G$ be a graded Lie group, i.e. a connected simply connected Lie group such that its Lie algebra $\mathfrak{g}$ has a vector space decomposition

$$
\mathfrak{g}=\bigoplus_{j=1}^{\infty} V_{j},
$$

such that all but finitely many of the $V_{j}$ 's are $\{0\}$ and $\left[V_{i}, V_{j}\right] \subset V_{i+j}$. A special case analysed in detail by Folland [21] is of stratified Lie groups when the first stratum $V_{1}$ generates $\mathfrak{g}$ as an algebra, see also Folland and Stein [23]. A typical example of such Lie group is the Heisenberg group. In general, graded Lie groups are necessarily homogeneous and nilpotent. Moreover, any graded Lie group can be viewed as some $\mathbb{R}^{n}$ with a polynomial group law. We can also refer to [18, Sect. 3.1] for a detailed discussion of graded Lie groups and their properties.

Let $\mathcal{R}$ be a positive Rockland operator on $G$, that is, a positive (in the operator sense) leftinvariant differential operator which is homogeneous of degree $v>0$ and which satisfies the so-called Rockland condition. This means that for each representation $\pi \in \widehat{G}$, except for the trivial one, the operator $\pi(\mathcal{R})$ is injective on the space of smooth vectors $\mathcal{H}_{\pi}^{\infty}$, i.e.

$$
\forall v \in \mathcal{H}_{\pi}^{\infty} \quad \pi(\mathcal{R}) v=0 \Longrightarrow v=0 .
$$

Alternative characterisations of such operators have been considered by Rockland [40] and Beals [1], until the definitive result of Helffer and Nourrigat [29] saying that Rockland operators are precisely the left-invariant homogeneous hypoelliptic differential operators on $G$. The existence of Rockland operators on general nilpotent Lie groups characterises precisely the class of graded Lie groups $[35,46]$. An example of a positive Rockland operator is the positive sub-Laplacian on a stratified Lie group: if $G$ is a stratified Lie group and 
$\left\{X_{1}, \ldots, X_{k}\right\}$ is a basis for the first stratum of its Lie algebra, then the positive sub-Laplacian

$$
\mathcal{L}=-\sum_{j=1}^{k} X_{j}^{2}
$$

is a positive Rockland operator. Moreover, for any $m \in \mathbb{N}$, the operator

$$
\mathcal{R}=(-1)^{m} \sum_{j=1}^{k} X_{j}^{2 m}
$$

is a positive Rockland operator on the stratified Lie group $G$. More generally, for any graded Lie group $G \sim \mathbb{R}^{n}$, if $X_{1}, \ldots, X_{n}$ is the basis of its Lie algebra $\mathfrak{g}$ with dilation weights $v_{1}, \ldots, v_{n}$, i.e. with

$$
D_{r} X_{j}=r^{v_{j}} X_{j}, \quad j=1, \ldots, n, r>0,
$$

where $D_{r}$ are dilations on $\mathfrak{g}$, then the operator

$$
\mathcal{R}=\sum_{j=1}^{n}(-1)^{\frac{\nu_{0}}{v_{j}}} a_{j} X_{j}^{2 \frac{v_{0}}{v_{j}}}, \quad a_{j}>0,
$$

is a Rockland operator of homogeneous degree $2 v_{0}$, if $v_{0}$ is any common multiple of $v_{1}, \ldots, v_{n}$. We refer to [18, Sect. 4.1.2] for other examples and a detailed discussion of Rockland operators and graded Lie groups. In the case of $\mathbb{R}^{n}$, all elliptic homogeneous differential operators with constant coefficients are Rockland operators.

To formulate our results we will need two scales of spaces, namely, Sobolev and Gevrey spaces, adapted to the setting of graded Lie groups. Thus, let $G$ be a graded Lie group and let $\mathcal{R}$ be a positive Rockland operator of homogeneous degree $v$. For any real number $s \in \mathbb{R}$, the Sobolev space $H_{\mathcal{R}}^{s}(G)$ is the subspace of $\mathcal{S}^{\prime}(G)$ obtained as the completion of the Schwartz space $\mathcal{S}(G)$ with respect to the Sobolev norm

$$
\|f\|_{H_{\mathcal{R}}^{s}(G)}:=\left\|(I+\mathcal{R})^{\frac{s}{v}} f\right\|_{L^{2}(G)} .
$$

For stratified Lie groups such spaces and their properties have been extensively analysed by Folland in [21] and on general graded Lie groups they have been investigated in $[18,19]$. In particular, these spaces do not depend on a particular choice of the Rockland operator $\mathcal{R}$ used in the definition (1.5), see [18, Theorem 4.4.20]. These spaces perfectly suit Case 1 described above but already in the Euclidean case, with the elliptic Laplace operator instead of the hypoelliptic Rockland operator in the wave equation (1.1), if the coefficient $a(t)$ is not Lipschitz regular or may become zero, the Gevrey spaces appear naturally (see e.g. Bronshtein [3]) since we cannot expect anymore the well-posedness in $\mathcal{C}^{\infty}(G)$ or $\mathcal{D}^{\prime}(G)$. Indeed, Colombini and Spagnolo exhibited a concrete example in [11] of a Cauchy problem for the time-dependent wave equation on $\mathbb{R}$ with smooth $a \geq 0$ which is not well-posed in $\mathcal{C}^{\infty}(\mathbb{R})$ or $\mathcal{D}^{\prime}(\mathbb{R})$.

Thus, given $s \geq 1$, we define the Gevrey type space

$$
\gamma_{\mathcal{R}}^{s}(G):=\left\{f \in \mathcal{C}^{\infty}(G) \mid \exists A>0:\left\|e^{A \mathcal{R} \frac{1}{2 s}} f\right\|_{L^{2}(G)}<\infty\right\} .
$$

These spaces provide a subelliptic version of the usual Gevrey spaces. For example, for $G=\mathbb{H}^{n}$ being the Heisenberg group with the basis $X_{1}, \ldots, X_{2 n}$ of the first stratum, it was 
shown in [20] that $f \in \gamma_{\mathcal{R}}^{s}\left(\mathbb{H}^{n}\right)$ if and only if there exist two constants $B, C>0$ such that for every $\alpha \in \mathbb{N}_{0}^{2 n}$ the following inequality holds

$$
\left\|\partial^{\alpha} f\right\|_{L^{2}\left(\mathbb{H}^{n}\right)} \leq C B^{|\alpha|}(\alpha !)^{s},
$$

where $\partial^{\alpha}=Y_{1} \ldots Y_{|\alpha|}$, with $Y_{j} \in\left\{X_{1}, \ldots, X_{2 n}\right\}$ for every $j=1, \ldots,|\alpha|$ and $\sum_{Y_{j}=X_{k}} 1=\alpha_{k}$ for every $k=1, \ldots, 2 n$.

Gevrey spaces (1.6) and the corresponding spaces of ultradistributions have been considered on compact Lie groups and on compact manifolds in [16] and in [17], respectively.

By an argument similar to that in [20] for the sub-Laplacian or in [16, Theorem 2.4] for elliptic operators, it can be shown that if $\mathcal{R}$ is a positive Rockland operator of homogeneous degree $v$, then $f \in \gamma_{\mathcal{R}}^{s}(G)$ if and only if there exist constants $B, C>0$ such that for every $k \in \mathbb{N} \cup\{0\}$ we have

$$
\left\|\mathcal{R}^{k} f\right\|_{L^{2}(G)} \leq C B^{\nu k}((\nu k) !)^{s} .
$$

Since Sobolev spaces do not depend on a particular choice of the Rockland operator used in their definition, the characterisation (1.8) of the Gevrey spaces implies that the same is true for $\gamma_{\mathcal{R}}^{s}(G)$.

Thus, we may drop the subscript $\mathcal{R}$ in $H_{\mathcal{R}}^{s}$ and $\gamma_{\mathcal{R}}^{s}$ but we may also keep using it to refer to the norms that we may be using.

Let us now formulate the main theorem of our paper, where we consider the following four cases:

Case 1: $a \in \operatorname{Lip}([0, T]), a(t) \geq a_{0}>0$;

Case 2: $a \in \mathcal{C}^{\alpha}([0, T]), 0<\alpha<1, a(t) \geq a_{0}>0$;

Case 3: $a \in \mathcal{C}^{l}([0, T]), l \geq 2, a(t) \geq 0$;

Case 4: $a \in \mathcal{C}^{\alpha}([0, T])$, with $0<\alpha<2, a(t) \geq 0$.

These are the four cases to which we refer repeatedly throughout this paper.

Theorem 1.1 Let $G$ be a graded Lie group and let $\mathcal{R}$ be a positive Rockland operator of homogeneous degree $v$. Let $T>0$. Then the following holds, referring respectively to Cases 1-4 above:

Case 1: Given $s \in \mathbb{R}$, if the initial Cauchy data $\left(u_{0}, u_{1}\right)$ are in $H_{\mathcal{R}}^{s+\frac{v}{2}}(G) \times H_{\mathcal{R}}^{s}(G)$, then there exists a unique solution of $(1.1)$ in the space $\mathcal{C}\left([0, T], H_{\mathcal{R}}^{s+\frac{v}{2}}(G)\right) \cap$ $\mathcal{C}^{1}\left([0, T], H_{\mathcal{R}}^{s}(G)\right)$, satisfying the following inequality for all values of $t \in[0, T]$ :

$$
\|u(t, \cdot)\|_{H_{\mathcal{R}}^{s+\frac{\nu}{2}}}^{2}+\left\|\partial_{t} u(t, \cdot)\right\|_{H_{\mathcal{R}}^{s}}^{2} \leq C\left(\left\|u_{0}\right\|_{H_{\mathcal{R}}^{s+\frac{v}{2}}}^{2}+\left\|u_{1}\right\|_{H_{\mathcal{R}}^{s}}^{2}\right)
$$

Case 2: If the initial Cauchy data $\left(u_{0}, u_{1}\right)$ are in $\gamma_{\mathcal{R}}^{s}(G) \times \gamma_{\mathcal{R}}^{s}(G)$, then there exists a unique solution of $(1.1)$ in $\mathcal{C}^{2}\left([0, T], \gamma_{\mathcal{R}}^{s}(G)\right)$, provided that

$$
1 \leq s<1+\frac{\alpha}{1-\alpha}
$$

Case 3: If the initial Cauchy data $\left(u_{0}, u_{1}\right)$ are in $\gamma_{\mathcal{R}}^{s}(G) \times \gamma_{\mathcal{R}}^{s}(G)$, then there exists a unique solution of $(1.1)$ in $\mathcal{C}^{2}\left([0, T], \gamma_{\mathcal{R}}^{s}(G)\right)$, provided that

$$
1 \leq s<1+\frac{l}{2}
$$


Case 4: If the initial Cauchy data $\left(u_{0}, u_{1}\right)$ are in $\gamma_{\mathcal{R}}^{s}(G) \times \gamma_{\mathcal{R}}^{s}(G)$ then there exists a unique solution of $(1.1)$ in $\mathcal{C}^{2}\left([0, T], \gamma_{\mathcal{R}}^{s}(G)\right)$, provided that

$$
1 \leq s<1+\frac{\alpha}{2} .
$$

As it will follow from the proof, in Cases 2 and 4, one can take the equalities $s=1+\frac{\alpha}{1-\alpha}$ and $s=1+\frac{\alpha}{2}$, respectively, provided that $T>0$ is small enough. We refer to $[25,26]$ concerning the sharpness of the above Gevrey indices in the case of $G=\mathbb{R}^{n}$ and $\mathcal{R}=-\Delta$, and for further relevant references for that case.

Let us formulate a corollary from Theorem 1.1 showing the local loss of regularity for the Cauchy problem (1.1). We recall that any graded Lie group $G$ can be identified, for example through the exponential mapping, with the Euclidean space $\mathbb{R}^{n}$ where $n$ is the topological dimension of $G$. Then, if $v_{1}, \ldots, v_{n}$ are the dilation weights on $G$ as in (1.4), for any $s \in \mathbb{R}$ we have the local Sobolev embedding theorems:

$$
H_{l o c}^{s / \nu_{1}}\left(\mathbb{R}^{n}\right) \subset H_{\mathcal{R}, l o c}^{s}(G) \subset H_{l o c}^{s / v_{n}}\left(\mathbb{R}^{n}\right),
$$

see [18, Theorem 4.4.24]. If $G$ is a stratified Lie group, we have $v_{1}=1$ and $v_{n}$ is the step of $G$, i.e. the number of steps in the stratification of its Lie algebra. In other words, if $G$ is a stratified Lie group of step $r$ and $H^{s}(G)$ is the Sobolev space defined using (any) subLaplacian on $G$, then the embeddings (1.10) are reduced to

$$
H_{l o c}^{s}\left(\mathbb{R}^{n}\right) \subset H_{l o c}^{s}(G) \subset H_{l o c}^{s / r}\left(\mathbb{R}^{n}\right) .
$$

These embeddings are sharp, see Folland [21]. Consequently, using the characterisation (1.8) of $\gamma_{\mathcal{R}}^{s}(G)$, we also obtain the embeddings

$$
\gamma_{l o c}^{\nu_{1} s}\left(\mathbb{R}^{n}\right) \subset \gamma_{\mathcal{R}, l o c}^{s}(G) \subset \gamma_{l o c}^{s \nu_{n}}\left(\mathbb{R}^{n}\right),
$$

where the space $\gamma_{l o c}^{\sigma}\left(\mathbb{R}^{n}\right)$ is the usual Euclidean Gevrey space, namely, the space of all smooth functions $f \in \mathcal{C}^{\infty}\left(\mathbb{R}^{n}\right)$ such that for every compact set $K \subset \mathbb{R}^{n}$ there exist two constants $B, C>0$ such that for every $\alpha$ we have

$$
\left|\partial^{\alpha} f(x)\right| \leq C B^{|\alpha|}(\alpha !)^{\sigma} \quad \text { for all } x \in K .
$$

Hence, if $G$ is a stratified Lie group of step $r$ we have the embeddings

$$
\gamma_{l o c}^{s}\left(\mathbb{R}^{n}\right) \subset \gamma_{\mathcal{R}, l o c}^{s}(G) \subset \gamma_{l o c}^{s r}\left(\mathbb{R}^{n}\right) .
$$

Consequently, using these embeddings, we obtain the following local in space wellposedness result using the usual Euclidean Gevrey spaces. Here we may also assume that the Cauchy data are compactly supported due to the finite propagation speed of singularities. To emphasise the appearing phenomenon of local loss of Euclidean regularity we formulate it in the simplified setting of stratified Lie groups, with topological identification $G \sim \mathbb{R}^{n}$. The spaces $\gamma^{s}\left(\mathbb{R}^{n}\right)$ below denote the usual Gevrey spaces on $\mathbb{R}^{n}$.

Corollary 1.2 Let $G \sim \mathbb{R}^{n}$ be a stratified Lie group of step $r$ and let $\mathcal{R}$ be a positive Rockland operator of homogeneous degree $v$ (for example, $\mathcal{R}$ can be a positive sub-Laplacian in which case we have $v=2)$. Assume that the Cauchy data $\left(u_{0}, u_{1}\right)$ are compactly supported. Then the following holds, referring respectively to Cases 1-4 above: 
Case 1: Given $s \in \mathbb{R}$, if $\left(u_{0}, u_{1}\right)$ are in $H^{s+\frac{v}{2}}\left(\mathbb{R}^{n}\right) \times H^{s}\left(\mathbb{R}^{n}\right)$, then there exists a unique solution of (1.1) in $\mathcal{C}\left([0, T], H^{\left(s+\frac{v}{2}\right) / r}\left(\mathbb{R}^{n}\right)\right) \cap \mathcal{C}^{1}\left([0, T], H^{s / r}\left(\mathbb{R}^{n}\right)\right)$, satisfying the following inequality for all values of $t \in[0, T]$ :

$$
\|u(t, \cdot)\|_{H^{\left(s+\frac{v}{2}\right) / r}}^{2}+\left\|\partial_{t} u(t, \cdot)\right\|_{H^{s / r}}^{2} \leq C\left(\left\|u_{0}\right\|_{H^{s+\frac{\nu}{2}}}^{2}+\left\|u_{1}\right\|_{H^{s}}^{2}\right)
$$

Case 2: If $\left(u_{0}, u_{1}\right)$ are in $\gamma^{s}\left(\mathbb{R}^{n}\right) \times \gamma^{s}\left(\mathbb{R}^{n}\right)$, then there exists a unique solution of (1.1) in $\mathcal{C}^{2}\left([0, T], \gamma^{s r}\left(\mathbb{R}^{n}\right)\right)$, provided that

$$
1<s<1+\frac{\alpha}{1-\alpha}
$$

Case 3: If $\left(u_{0}, u_{1}\right)$ are in $\gamma^{s}\left(\mathbb{R}^{n}\right) \times \gamma^{s}\left(\mathbb{R}^{n}\right)$, then there exists a unique solution of (1.1) in $\mathcal{C}^{2}\left([0, T], \gamma^{s r}\left(\mathbb{R}^{n}\right)\right)$, provided that

$$
1<s<1+\frac{l}{2}
$$

Case 4: If $\left(u_{0}, u_{1}\right)$ are in $\gamma^{s}\left(\mathbb{R}^{n}\right) \times \gamma^{s}\left(\mathbb{R}^{n}\right)$ then there exists a unique solution of (1.1) in $\mathcal{C}^{2}\left([0, T], \gamma^{s r}\left(\mathbb{R}^{n}\right)\right)$, provided that

$$
1<s<1+\frac{\alpha}{2}
$$

The statements in Cases 2-4 for $s=1$ are not so interesting, with the analytic wellposedness known in these case anyway, see Bony and Shapira [2].

For $G=\mathbb{R}^{n}$ and $\mathcal{R}$ being the Laplacian, we have $r=1$ and there is no loss of regularity in any of the Cases 1-4, when the results are known from [7, 12, 25, 26, 33].

We remark that in the meantime, also models with more singular coefficients have been treated in the context of very weak solutions, see [44].

However, already on the Heisenberg group with step $r=2$, we observe the local loss of regularity in Euclidean Sobolev and Gevrey spaces in all statements of Cases 1-4 in Corollary 1.2.

We also note that using local Sobolev and Gevrey embeddings (1.10) and (1.12), it is easy to formulate an extension of Corollary 1.2 to general graded Lie groups.

\section{Preliminaries on Graded Lie Groups and Rockland Operators}

In this section we recall some preliminaries and fix the notation concerning the Fourier analysis on graded Lie groups. We refer to [24] and to [18, Chap. 5] for further details.

Thus, a connected and simply connected Lie group $G$ is called graded when its Lie algebra is graded in the sense of the decomposition (1.2).

A Lie algebra $\mathfrak{g}$ is stratified if it is graded and if its first stratum $V_{1}$ generates $\mathfrak{g}$ as an algebra. Thus, in this case every element of the Lie algebra can be written as a linear combination of elements in $V_{1}$ and their iterated commutators. A Lie group is stratified when it is connected, simply connected and its Lie algebra is stratified.

Furthermore, if there are $r$ non zero $V_{j}$ 's in the vector space decomposition (1.2), then the group (respectively the algebra) is said to be stratified of step $r$. 
From the definition of a stratified Lie algebra, it follows that, assuming that $V_{1}$ has dimension $k$, any basis $\left\{X_{1}, \ldots, X_{k}\right\}$ for $V_{1}$ forms a Hörmander system, see [31], and we can consider its associated sub-Laplacian operator that is also a positive Rockland operator:

$$
\mathcal{L}:=-\left(X_{1}^{2}+\cdots+X_{k}^{2}\right) .
$$

Example 2.1 (The Heisenberg group) A classical example of a graded (stratified) Lie group is the Heisenberg group $\mathbb{H}^{n}$ that might be seen as the manifold $\mathbb{R}^{2 n+1}$ endowed with the group law

$$
(x, y, t)\left(x^{\prime}, y^{\prime}, t^{\prime}\right):=\left(x+x^{\prime}, y+y^{\prime}, t+t^{\prime}+\frac{1}{2}\left(x \cdot y^{\prime}-x^{\prime} \cdot y\right)\right)
$$

where $(x, y, t),\left(x^{\prime}, y^{\prime}, t^{\prime}\right) \in \mathbb{R}^{n} \times \mathbb{R}^{n} \times \mathbb{R} \sim \mathbb{H}^{n}$. The Heisenberg Lie algebra $\mathfrak{h}^{n}$ associated with the Heisenberg group is the space of all the left-invariant vector fields of $\mathbb{H}^{n}$ and it admits the following canonical basis:

$$
\begin{aligned}
& X_{j}=\partial_{x_{j}}-\frac{y_{j}}{2} \partial_{t}, \\
& Y_{j}=\partial_{y_{j}}+\frac{x_{j}}{2} \partial_{t}, \\
& T=\partial_{t} .
\end{aligned}
$$

The former vector fields satisfy the canonical commutation relations

$$
\left[X_{j}, Y_{j}\right]=T, \quad \forall j=1, \ldots, n,
$$

and all the other possible commutators are zero. Therefore, the Heisenberg group is a graded (stratified) Lie group of step 2, whose Lie algebra admits the vector space decomposition

$$
\mathfrak{h}^{n}=V_{1} \oplus V_{2},
$$

where

$$
V_{1}=\sum_{j=1}^{n} \mathbb{R} X_{j} \oplus \mathbb{R} Y_{j} \quad \text { and } \quad V_{2}=\mathbb{R} T .
$$

Hypoellipticity and other questions on the Heisenberg group have a long history, see e.g. Taylor [45], Folland [22], or Thangavelu [47], and many references therein.

From now on, we consider $G$ to be a graded Lie group, even if some of the following definitions and remarks hold in a more general setting.

Let $\pi$ be a representation of $G$ on the separable Hilbert space $\mathcal{H}_{\pi}$. A vector $v \in \mathcal{H}_{\pi}$ is said to be smooth or of type $\mathcal{C}^{\infty}$ if the function

$$
G \ni x \mapsto \pi(x) v \in \mathcal{H}_{\pi}
$$

is of class $\mathcal{C}^{\infty}$. The space of all smooth vectors of a representation $\pi$ is denoted by $\mathcal{H}_{\pi}^{\infty}$. Let $\mathfrak{g}$ be the Lie algebra of $G$ and let $\pi$ be a strongly continuous representation of $G$ on a Hilbert space $\mathcal{H}_{\pi}$. For every $X \in \mathfrak{g}$ and $v \in \mathcal{H}_{\pi}^{\infty}$ we define

$$
d \pi(X) v:=\lim _{t \rightarrow 0} \frac{1}{t}\left(\pi\left(\exp _{G}(t X)\right) v-v\right) .
$$


Then $d \pi$ is a representation of $\mathfrak{g}$ on $\mathcal{H}_{\pi}^{\infty}$ (see e.g. [18, Proposition 1.7.3]) called the infinitesimal representation associated to $\pi$. By abuse of notation, we will often still denote it by $\pi$, therefore, for any $X \in \mathfrak{g}$, we write $\pi(X)$ meaning $d \pi(X)$.

Any left-invariant differential operator $T$ on $G$, according to the Poincaré-Birkhoff-Witt theorem, can be written in a unique way as a finite sum

$$
T=\sum_{|\alpha| \leq M} c_{\alpha} X^{\alpha}
$$

where all but finitely many of the coefficients $c_{\alpha} \in \mathbb{C}$ are zero and $X^{\alpha}=X_{1} \ldots X_{|\alpha|}$, with $X_{j} \in \mathfrak{g}$. This allows one to look at any left-invariant differential operator $T$ on $G$ as an element of the universal enveloping algebra $\mathfrak{U}(\mathfrak{g})$ of the Lie algebra of $G$. Therefore, the family of infinitesimal representations $\{\pi(T), \pi \in \widehat{G}\}$ yields a field of operators that turns to be the symbol associated with the operator $T$.

Let $\pi \in \widehat{G}$ and let $\mathcal{R}$ be a positive Rockland operator of homogeneous degree $v>0$, then using formula (2.2), the infinitesimal representation of $\mathcal{R}$ associated to $\pi$ is

$$
\pi(\mathcal{R})=\sum_{[\alpha]=\nu} c_{\alpha} \pi(X)^{\alpha},
$$

where $\pi(X)^{\alpha}=\pi\left(X^{\alpha}\right)=\pi\left(X_{1}^{\alpha_{1}} \cdots X_{n}^{\alpha_{n}}\right)$ and $[\alpha]=v_{1} \alpha_{1}+\cdots+v_{n} \alpha_{n}$ is the homogeneous degree of the multiindex $\alpha$, with $X_{j}$ being homogeneous of degree $v_{j}$.

The operator $\mathcal{R}$ and its infinitesimal representations $\pi(\mathcal{R})$ are densely defined on $\mathcal{D}(G) \subset L^{2}(G)$ and $\mathcal{H}_{\pi}^{\infty} \subset \mathcal{H}_{\pi}$, respectively, see e.g. [18, Proposition 4.1.15]. We denote by $\mathcal{R}_{2}$ the self-adjoint extension of $\mathcal{R}$ on $L^{2}(G)$ and we keep the same notation $\pi(\mathcal{R})$ for the self-adjoint extensions on $\mathcal{H}_{\pi}$ of the infinitesimal representations. Recalling the spectral theorem for unbounded operators [39, Theorem VIII.6], we can consider the spectral measures $E$ and $E_{\pi}$ corresponding to $\mathcal{R}_{2}$ and $\pi(\mathcal{R})$, so that we have

$$
\mathcal{R}_{2}=\int_{\mathbb{R}} \lambda d E(\lambda) \quad \text { and } \quad \pi(\mathcal{R})=\int_{\mathbb{R}} \lambda d E_{\pi}(\lambda) .
$$

Furthermore, for any $f \in L^{2}(G)$ we have

$$
\mathcal{F}(\phi(\mathcal{R}) f)(\pi)=\phi(\pi(\mathcal{R})) \widehat{f}(\pi)
$$

for any measurable bounded function $\phi$ on $\mathbb{R}$, see e.g. [18, Corollary 4.1.16]. The infinitesimal representations $\pi(\mathcal{R})$ of a positive Rockland operator are also positive, due to the relations between their spectral measures. In particular, Hulanicki, Jenkins and Ludwig showed in [32] that the spectrum of $\pi(\mathcal{R})$, with $\pi \in \widehat{G} \backslash\{1\}$, is discrete and lies in $(0, \infty)$. This implies that we can choose an orthonormal basis for $\mathcal{H}_{\pi}$ such that the infinite matrix associated to the self-adjoint operator $\pi(\mathcal{R})$ has the form

$$
\pi(\mathcal{R})=\left(\begin{array}{cccc}
\pi_{1}^{2} & 0 & \ldots & \ldots \\
0 & \pi_{2}^{2} & 0 & \ldots \\
\vdots & 0 & \ddots & \\
\vdots & \vdots & & \ddots
\end{array}\right),
$$

where $\pi_{j}$ are strictly positive real numbers and $\pi \in \widehat{G} \backslash\{1\}$. 


\section{Parameter Dependent Energy Estimates}

In this section we prove certain energy estimates for second order ordinary differential equations with explicit dependence on parameters. This will be crucial in the proof of Theorem 1.1 where the parameters will correspond to the spectral decomposition (2.4) of the infinitesimal representations of the Rockland operators.

Results of the following type have been of use in different estimates related to weakly hyperbolic partial differential equations, such as [7] and [27]. However, in those papers the conclusions rely on more general results, see [25]. We partly follow the argument in [27] based on a standard reduction to a first order system. Consequently, we carry out different types of arguments depending on assumptions in each of the cases, altogether allowing us to formulate the precise dependence on parameters for ordinary differential equations corresponding to the propagation coefficient $a(t)$ as in Cases 1-4 of Theorem 1.1, to which we refer in the following statement.

Proposition 3.1 Let $\beta>0$ be a positive constant and let $a(t)$ be a function that behaves according to Cases 1, 2, 3 and 4 in Theorem 1.1. Let $T>0$. Consider the following Cauchy problem:

$$
\left\{\begin{array}{l}
v^{\prime \prime}(t)+\beta^{2} a(t) v(t)=0 \quad \text { with } t \in(0, T] \\
v(0)=v_{0} \in \mathbb{C} \\
v^{\prime}(0)=v_{1} \in \mathbb{C}
\end{array}\right.
$$

Then the following holds:

Case 1: There exists a positive constant $C>0$ such that for all $t \in[0, T]$ we have

$$
\beta^{2}|v(t)|^{2}+\left|v^{\prime}(t)\right|^{2} \leq C\left(\beta^{2}\left|v_{0}\right|^{2}+\left|v_{1}\right|^{2}\right) .
$$

Case 2: There exist two positive constants $C, K>0$ such that for all $t \in[0, T]$ we have

$$
\beta^{2}|v(t)|^{2}+\left|v^{\prime}(t)\right|^{2} \leq C e^{K t \beta^{\frac{1}{s}}}\left(\beta^{2}\left|v_{0}\right|^{2}+\left|v_{1}\right|^{2}\right),
$$

for any $1 \leq s<1+\frac{\alpha}{1-\alpha}$. Moreover, there exists a constant $k>0$ such that for any $\beta_{0} \geq 1$ the estimate (3.2) holds for $K=k \beta_{0}^{1-\alpha-\frac{1}{s}}$ for all $\beta \geq \beta_{0}$.

Case 3: There exist two positive constants $C, K>0$ such that for all $t \in[0, T]$ we have

$$
\beta^{2}|v(t)|^{2}+\left|v^{\prime}(t)\right|^{2} \leq C\left(1+\beta^{\frac{l}{\sigma}}\right) e^{K \beta^{\frac{1}{\sigma}}}\left(\beta^{2}\left|v_{0}\right|^{2}+\left|v_{1}\right|^{2}\right),
$$

with $\sigma=1+\frac{l}{2}$.

Case 4: There exist two positive constants $C, K>0$ such that

$$
\beta^{2}|v(t)|^{2}+\left|v^{\prime}(t)\right|^{2} \leq C\left(1+\beta^{\frac{\alpha / 2}{\alpha / 2+1}}\right) e^{K t \beta^{\frac{1}{s}}}\left(\beta^{2}\left|v_{0}\right|^{2}+\left|v_{1}\right|^{2}\right),
$$

for any $1 \leq s<1+\frac{\alpha}{2}$. Moreover, there exists a constant $k>0$ such that for any $\beta_{0} \geq 1$ the estimate (3.3) holds for $K=k \beta_{0}^{\frac{1}{1+\alpha / 2}-\frac{1}{s}}$ for all $\beta \geq \beta_{0}$.

The constants $C$ in the above inequalities may depend on $T$ but not on $\beta$. 
Proof First we reduce the problem (3.1) to a first order system. In order to do this we rewrite it in a standard way as a matrix-valued equation. Thus we define the column vectors

$$
V(t):=\left(\begin{array}{c}
i \beta v(t) \\
\partial_{t} v(t)
\end{array}\right), \quad V_{0}:=\left(\begin{array}{c}
i \beta v_{0} \\
v_{1}
\end{array}\right)
$$

and the matrix

$$
A(t):=\left(\begin{array}{cc}
0 & 1 \\
a(t) & 0
\end{array}\right)
$$

that allow us to reformulate the second order system (3.1) as the first order system

$$
\left\{\begin{array}{l}
V_{t}(t)=i \beta A(t) V(t) \\
V(0)=V_{0}
\end{array}\right.
$$

We will now treat each case separately.

\subsection{Case 1: $a \in \operatorname{Lip}([0, T]), a(t) \geq a_{0}>0$}

This is the simplest case that can be treated by a classical argument. We observe that the eigenvalues of our matrix $A(t)$ are given by $\pm \sqrt{a(t)}$. The symmetriser $S$ of $A$, i.e. the matrix such that

$$
S A-A^{*} S=0
$$

is given by

$$
S(t)=\left(\begin{array}{cc}
2 a(t) & 0 \\
0 & 2
\end{array}\right)
$$

Thus we define the energy as

$$
E(t):=(S(t) V(t), V(t))
$$

and we want to estimate its variations in time. A straightforward calculation yields the following inequality that will help us to get such estimate:

$$
2|V|^{2} \min _{t \in[0, T]}\{a(t), 1\} \leq E(t) \leq 2|V|^{2} \max _{t \in[0, T]}\{a(t), 1\} .
$$

In particular, in this case the continuity of $a(t)$ ensures the existence of two strictly positive constants $a_{0}$ and $a_{1}$ such that

$$
a_{0}=\min _{t \in[0, T]} a(t) \quad \text { and } \quad a_{1}=\max _{t \in[0, T]} a(t) .
$$

Thus setting $c_{0}:=2 \min \left\{a_{0}, 1\right\}$ and $c_{1}:=2 \max \left\{a_{1}, 1\right\}$, the inequality (3.5) becomes

$$
c_{0}|V(t)|^{2} \leq E(t) \leq c_{1}|V(t)|^{2} .
$$

A straightforward calculation, together with (3.6), gives the following estimate:

$$
\begin{aligned}
E_{t}(t) & =\left(S_{t}(t) V(t), V(t)\right)+\left(S(t) V_{t}(t), V(t)\right)+\left(S(t) V(t), V_{t}(t)\right)= \\
& =\left(S_{t}(t) V(t), V(t)\right)+i \beta(S(t) A(t) V(t), V(t))-i \beta(S(t) V(t), A(t) V(t))=
\end{aligned}
$$




$$
\begin{aligned}
& =\left(S_{t}(t) V(t), V(t)\right)+i \beta\left(\left(S(t) A(t)-A^{*}(t) S(t)\right) V(t), V(t)\right)= \\
& =\left(S_{t}(t) V(t), V(t)\right) \leq\left\|S_{t}(t)\right\||V(t)|^{2},
\end{aligned}
$$

thus setting $c^{\prime}:=c_{0}^{-1} \sup _{t \in[0, T]}\left\|S_{t}(t)\right\|$, we get from (3.7) using (3.6) that

$$
E_{t}(t) \leq c^{\prime} E(t)
$$

Applying the Gronwall lemma to (3.8), we deduce that there exists a constant $c>0$ independent of $t \in[0, T]$ such that

$$
E(t) \leq c E(0)
$$

Therefore, putting together (3.9) and (3.6) we obtain

$$
c_{0}|V(t)|^{2} \leq E(t) \leq c E(0) \leq c c_{1}|V(0)|^{2} .
$$

We can then rephrase this, asserting that there exists a constant $C>0$ independent of $t$ such that $|V(t)|^{2} \leq C|V(0)|^{2}$. Then we write this inequality going back to the definition of $V(t)$, yielding

$$
\beta^{2}|v(t)|^{2}+\left|\partial_{t} v(t)\right|^{2} \leq C\left(\beta^{2}\left|v_{0}\right|^{2}+\left|v_{1}\right|^{2}\right)
$$

as required.

\subsection{Case 2: $a \in \mathcal{C}^{\alpha}([0, T])$, with $0<\alpha<1, a(t) \geq a_{0}>0$}

Here we follow the method developed by Colombini and Kinoshita [7] for $n=1$ and subsequently extended [25] for any $n \in \mathbb{N}$. We look for solutions of the form

$$
V(t)=e^{-\rho(t) \beta^{\frac{1}{s}}}(\operatorname{det} H(t))^{-1} H(t) W(t),
$$

where

- $s \in \mathbb{R}$ depends on $\alpha$ as will be determined in the argument;

- the function $\rho=\rho(t) \in \mathcal{C}^{1}([0, T])$ is real-valued and will be chosen later;

- $W(t)$ is the energy;

- $H(t)$ is the matrix defined by

$$
H(t):=\left(\begin{array}{cc}
1 & 1 \\
\lambda_{1}^{\epsilon}(t) & \lambda_{2}^{\epsilon}(t)
\end{array}\right)
$$

where for all $\epsilon>0, \lambda_{1}^{\epsilon}(t)$ and $\lambda_{2}^{\epsilon}(t)$ are regularisations of the eigenvalues of the matrix $A(t)$ of the form

$$
\begin{aligned}
& \lambda_{1}^{\epsilon}(t):=\left(-\sqrt{a} * \varphi_{\epsilon}\right)(t), \\
& \lambda_{2}^{\epsilon}(t):=\left(+\sqrt{a} * \varphi_{\epsilon}\right)(t),
\end{aligned}
$$

with $\left\{\varphi_{\epsilon}(t)\right\}_{\epsilon>0}$ being a family of cut-off functions defined starting from a non-negative function $\varphi \in \mathcal{C}_{c}^{\infty}(\mathbb{R})$, with $\int_{\mathbb{R}} \varphi=1$, by setting $\varphi_{\epsilon}(t):=\frac{1}{\epsilon} \varphi\left(\frac{t}{\epsilon}\right)$. By construction, it follows that $\lambda_{1}^{\epsilon}, \lambda_{2}^{\epsilon} \in \mathcal{C}^{\infty}([0, T])$. 
Furthermore, we can easily check, using the Hölder regularity of $a(t)$ of order $\alpha$ and, therefore, of $\sqrt{a(t)}$ of the same order $\alpha$, the following inequalities:

$$
\operatorname{det} H(t)=\lambda_{2}^{\epsilon}(t)-\lambda_{1}^{\epsilon}(t) \geq 2 \sqrt{a_{0}},
$$

and for all $t \in[0, T]$ and $\epsilon>0$ there exist two constants $c_{1}, c_{2}>0$ such that

$$
\begin{aligned}
& \left|\lambda_{1}^{\epsilon}(t)+\sqrt{a(t)}\right| \leq c_{1} \epsilon^{\alpha}, \\
& \left|\lambda_{2}^{\epsilon}(t)-\sqrt{a(t)}\right| \leq c_{2} \epsilon^{\alpha},
\end{aligned}
$$

uniformly in $t$ and $\epsilon$. Now we substitute our suggested solution (3.10) in (3.4) yielding

$$
\begin{aligned}
& -\rho^{\prime}(t) \beta^{\frac{1}{s}} e^{-\rho(t) \beta^{\frac{1}{s}}} \frac{H(t) W(t)}{\operatorname{det} H(t)}+e^{-\rho(t) \beta^{\frac{1}{s}}} \frac{H_{t}(t) W(t)}{\operatorname{det} H(t)}+e^{-\rho(t) \beta^{\frac{1}{s}}} \frac{H(t) W_{t}(t)}{\operatorname{det} H(t)}+ \\
& -e^{-\rho(t) \beta^{\frac{1}{s}}}(\operatorname{det} H)_{t}(t) \frac{H(t) W(t)}{(\operatorname{det} H(t))^{2}}=i \beta A(t) e^{-\rho(t) \beta^{\frac{1}{s}}} \frac{H(t) W(t)}{\operatorname{det} H(t)}
\end{aligned}
$$

Multiplying both sides of this equality by $e^{\rho(t) \beta^{\frac{1}{s}}} \operatorname{det} H(t) H^{-1}(t)$ we get

$$
\begin{aligned}
W_{t}(t) & =\rho^{\prime}(t) \beta^{\frac{1}{s}} W(t)-H^{-1}(t) H_{t}(t) W(t)+(\operatorname{det} H)_{t}(t)(\operatorname{det} H(t))^{-1} W(t)+ \\
& +i \beta H^{-1}(t) A(t) H(t) W(t) .
\end{aligned}
$$

This leads to the estimate

$$
\begin{aligned}
\frac{d}{d t}|W(t)|^{2} & =\left(W_{t}(t), W(t)\right)+\left(W(t), W_{t}(t)\right)=2 \operatorname{Re}\left(W_{t}(t), W(t)\right)= \\
& =2\left(\rho^{\prime}(t) \beta^{\frac{1}{s}}|W(t)|^{2}-\operatorname{Re}\left(H^{-1}(t) H_{t}(t) W(t), W(t)\right)+\right. \\
& \left.+(\operatorname{det} H(t))^{-1}(\operatorname{det} H)_{t}(t)|W(t)|^{2}+\beta \operatorname{Im}\left(H^{-1}(t) A(t) H(t) W(t), W(t)\right)\right) .
\end{aligned}
$$

We observe that

$$
\begin{aligned}
& 2 \operatorname{Im}\left(H^{-1} A H W, W\right)=\left(H^{-1} A H W, W\right)-\overline{\left(H^{-1} A H W, W\right)}= \\
& =\left(H^{-1} A H W, W\right)-\left(W, H^{-1} A H W\right)=\left(H^{-1} A H W, W\right)-\left(\left(H^{-1} A H\right)^{*} W, W\right)= \\
& =\left(\left(H^{-1} A H-\left(H^{-1} A H\right)^{*}\right) W, W\right) \leq\left\|H^{-1} A H-\left(H^{-1} A H\right)^{*}\right\||W|^{2} .
\end{aligned}
$$

Thus we obtain

$$
\begin{array}{r}
\frac{d}{d t}|W(t)|^{2} \leq\left(2 \rho^{\prime}(t) \beta^{\frac{1}{s}}+2\left\|H^{-1}(t) H_{t}(t)\right\|+2\left|(\operatorname{det} H(t))^{-1}(\operatorname{det} H)_{t}(t)\right|+\right. \\
\left.+\beta\left\|H^{-1} A H-\left(H^{-1} A H\right)^{*}\right\|\right)|W(t)|^{2} .
\end{array}
$$

To proceed we need to estimate the following quantities:

I) $\left\|H^{-1}(t) H_{t}(t)\right\|$;

II) $\left|(\operatorname{det} H(t))^{-1}(\operatorname{det} H)_{t}(t)\right|$;

III) $\left\|H^{-1} A H-\left(H^{-1} A H\right)^{*}\right\|$. 
In [25] and [7], the authors determined estimates for similar functions in a more general setting, i.e. starting from an equation of arbitrary order $m$. In this particular case, we can proceed by straightforward calculations without relying on the mentioned works.

We deal with these three terms as follows:

I) Since $H^{-1}(t)=\frac{1}{\lambda_{2}^{\epsilon}-\lambda_{1}^{\epsilon}}\left(\begin{array}{cc}\lambda_{2}^{\epsilon} & -1 \\ -\lambda_{1}^{\epsilon} & 1\end{array}\right)$ and $H_{t}(t)=\left(\begin{array}{cc}0 & 0 \\ \partial_{t} \lambda_{1}^{\epsilon} & \partial_{t} \lambda_{2}^{\epsilon}\end{array}\right)$, it follows that the entries of the matrix $H^{-1} H_{t}$ are given by the functions $\frac{\partial_{t} \lambda_{j}^{\epsilon}}{\lambda_{2}^{\epsilon}-\lambda_{1}^{\epsilon}}$. We have, for example for $\lambda_{2}$,

$$
\begin{aligned}
\partial_{t} \lambda_{2}^{\epsilon}(t) & =\sqrt{a} * \partial_{t} \varphi_{\epsilon}(t)=\frac{1}{\epsilon^{2}} \sqrt{a} * \varphi^{\prime}\left(\frac{t}{\epsilon}\right)=\frac{1}{\epsilon} \int \sqrt{a(t-\rho \epsilon)} \varphi^{\prime}(\rho) d \rho= \\
& =\frac{1}{\epsilon} \int(\sqrt{a(t-\rho \epsilon)}-\sqrt{a(t)}) \varphi^{\prime}(\rho) d \rho+\frac{1}{\epsilon} \sqrt{a(t)} \int \varphi^{\prime}(\rho) d \rho \leq k \epsilon^{\alpha-1},
\end{aligned}
$$

where we are using the Hölder continuity of $\sqrt{a}$ for the first term and the fact that the second term is zero, since $\int \varphi^{\prime}=0$. Combining the inequalities (3.11) and (3.15), we get for a suitable positive constant $k_{1}$ that

$$
\left\|H^{-1}(t) H_{t}(t)\right\| \leq k_{1} \epsilon^{\alpha-1} .
$$

II) First we can estimate

$$
\left|(\operatorname{det} H(t))^{-1}(\operatorname{det} H)_{t}(t)\right|=\frac{\partial_{t} \lambda_{2}^{\epsilon}-\partial_{t} \lambda_{1}^{\epsilon}}{\lambda_{2}^{\epsilon}-\lambda_{1}^{\epsilon}}=\frac{2 \partial_{t} \lambda_{2}^{\epsilon}}{\lambda_{2}^{\epsilon}-\lambda_{1}^{\epsilon}} \leq \frac{2 k \epsilon^{\alpha-1}}{2 \sqrt{a_{0}}},
$$

therefore,

$$
\left|(\operatorname{det} H(t))^{-1}(\operatorname{det} H)_{t}(t)\right| \leq k_{2} \epsilon^{\alpha-1},
$$

for a constant $k_{2}>0$.

III) Also in this case, we write explicitly the matrix we are interested in, that is

$$
H^{-1} A H-\left(H^{-1} A H\right)^{*}=\left(\begin{array}{cc}
0 & \frac{-2 a(t)+\left(\lambda_{1}^{\epsilon}\right)^{2}+\left(\lambda_{2}^{\epsilon}\right)^{2}}{\lambda_{1}^{\epsilon}-\lambda_{2}^{\epsilon}} \\
\frac{2 a(t)-\left(\left(\lambda_{1}^{\epsilon}\right)^{2}+\left(\lambda_{2}^{\epsilon}\right)^{2}\right)}{\lambda_{1}^{\epsilon}-\lambda_{2}^{\epsilon}} & 0
\end{array}\right) .
$$

Observing that, by definition, $\left(\lambda_{1}^{\epsilon}\right)^{2}=\left(\lambda_{2}^{\epsilon}\right)^{2}$, and recalling inequality (3.11), to get the desired norm estimate, it is enough to consider the function $\left|a(t)-\left(\lambda_{2}^{\epsilon}\right)^{2}\right|$. A straightforward calculation, using inequality (3.12), shows that

$$
\begin{aligned}
\left|a(t)-\left(\lambda_{2}^{\epsilon}\right)^{2}\right| & =\left|\left(\sqrt{a(t)}-\lambda_{2}^{\epsilon}\right)\left(\sqrt{a(t)}+\lambda_{2}^{\epsilon}\right)\right| \leq \\
& \leq c_{2} \epsilon^{\alpha}\left(\sqrt{a(t)}+\int \sqrt{a(t-s)} \varphi_{\epsilon}(s) d s\right)= \\
& =c_{2} \epsilon^{\alpha} \int(\sqrt{a(t)}+\sqrt{a(t-s \epsilon)}) \varphi(s) d s \leq 2 c_{2}\|\sqrt{a}\|_{L^{\infty}} \epsilon^{\alpha} .
\end{aligned}
$$

It follows that

$$
\left\|H^{-1} A H-\left(H^{-1} A H\right)^{*}\right\| \leq k_{3} \epsilon^{\alpha} .
$$


Going back to (3.14), combining it with estimates I), II) and III), we get an estimate for the derivative of the energy, that is

$$
\frac{d}{d t}|W(t)|^{2} \leq\left(2 \rho^{\prime}(t) \beta^{\frac{1}{s}}+2 k_{1} \epsilon^{\alpha-1}+2 k_{2} \epsilon^{\alpha-1}+k_{3} \beta \epsilon^{\alpha}\right)|W(t)|^{2} .
$$

At this point we choose $\epsilon=\frac{1}{\beta}$, observing that we can always consider $\beta$ large enough, say $\beta>1$, in order to have a small $\epsilon \in(0,1]$. Indeed, for $\beta \leq \beta_{0}$ for some fixed $\beta_{0}>0$, a modification of the argument below gives estimate (3.2) with constants depending only on $\beta_{0}$ and $T$. So we may assume that $\beta>\beta_{0}$ for $\beta_{0}$ to be specified. We define also $\rho(t):=$ $\rho(0)-K t$ for some $K>0$ to be specified. Substituting this in (3.16) we get for a suitable constant $k>0$ that

$$
\frac{d}{d t}|W(t)|^{2} \leq\left(2 \rho^{\prime}(t) \beta^{\frac{1}{s}}+2 k \beta^{1-\alpha}\right)|W(t)|^{2}=\left(-2 K+2 k \beta^{1-\alpha-\frac{1}{s}}\right) \beta^{\frac{1}{s}}|W(t)|^{2} .
$$

If we have

$$
\frac{1}{s}>1-\alpha \quad \Longleftrightarrow \quad s<1+\frac{\alpha}{1-\alpha},
$$

and then also

$$
K:=k \beta_{0}^{1-\alpha-\frac{1}{s}} \geq k \beta^{1-\alpha-\frac{1}{s}}
$$

then for all $t \in[0, T]$ we have

$$
\frac{d}{d t}|W(t)|^{2} \leq 0
$$

This monotonicity of the energy $W(t)$ yields the following boundedness for the solution vector $V(t)$ :

$$
\begin{aligned}
|V(t)| & =e^{-\rho(t) \beta^{\frac{1}{s}}}(\operatorname{det} H(t))^{-1}\|H(t)\||W(t)| \leq \\
& \leq e^{-\rho(t) \beta^{\frac{1}{s}}}(\operatorname{det} H(t))^{-1}\|H(t)\||W(0)|=e^{K t \beta^{\frac{1}{s}}} \frac{\operatorname{det} H(0)}{\operatorname{det} H(t)} \frac{\|H(t)\|}{\|H(0)\|}|V(0)| .
\end{aligned}
$$

Note that, according to property (3.11), the function $(\operatorname{det} H)^{-1}(t)$ is bounded. Furthermore, the behaviour of the convolution and the definition of $H(t)$ guarantee the existence of suitable constants $c, c^{\prime}>0$ such that $\|H(t)\| \leq c$ and $\left\|H^{-1}(0)\right\| \leq c^{\prime}$. Therefore, there exists a constant $C>0$ such that

$$
|V(t)| \leq C e^{K t \beta^{\frac{1}{s}}}|V(0)|
$$

that means, by definition of $V(t)$, that

$$
\beta^{2}|v(t)|^{2}+\left|v_{t}(t)\right| \leq C e^{K t \beta^{\frac{1}{s}}}\left(\beta^{2}\left|v_{0}\right|^{2}+\left|v_{1}\right|^{2}\right)
$$

proving the statement of Case 2. 


\subsection{Case 3: $a \in \mathcal{C}^{l}([0, T])$, with $l \geq 2, a(t) \geq 0$}

In this case we extend the technique developed for Case 1. First we perturb the symmetriser of the matrix $A(t)$. This is done considering the so-called quasi-symmetriser of $A(t)$, the idea introduced for such problems by D'Ancona and Spagnolo in [15].

Consider the quasi-symmetriser of $A(t)$, that is, a family of coercive, Hermitian matrices of the form

$$
Q_{\epsilon}^{(2)}(t):=S(t)+2 \epsilon^{2}\left(\begin{array}{ll}
1 & 0 \\
0 & 0
\end{array}\right)=\left(\begin{array}{cc}
2 a(t) & 0 \\
0 & 2
\end{array}\right)+2 \epsilon^{2}\left(\begin{array}{ll}
1 & 0 \\
0 & 0
\end{array}\right)
$$

for all $\epsilon \in(0,1]$, and such that $\left(Q_{\epsilon}^{(2)} A-A^{*} Q_{\epsilon}^{(2)}\right)$ goes to zero as $\epsilon$ goes to zero. The associated perturbed energy is given by

$$
E_{\epsilon}(t):=\left(Q_{\epsilon}^{(2)} V(t), V(t)\right)
$$

We proceed estimating the energy, calculating its derivatives in time, so that

$$
\begin{aligned}
& \frac{d}{d t} E_{\epsilon}(t)=\left(\frac{d}{d t} Q_{\epsilon}^{(2)}(t) V(t), V(t)\right)+\left(Q_{\epsilon}^{(2)} V_{t}(t), V(t)\right)+\left(Q_{\epsilon}^{(2)} V(t), V_{t}(t)\right)= \\
& =\left(\frac{d}{d t} Q_{\epsilon}^{(2)}(t) V(t), V(t)\right)+i \beta\left(\left(Q_{\epsilon}^{(2)} A(t)-A^{*}(t) Q_{\epsilon}^{(2)}\right) V(t), V(t)\right) .
\end{aligned}
$$

To estimate the second term in the right hand side, we set

$$
V(t)=\left(\begin{array}{c}
i \beta v(t) \\
\partial_{t} v
\end{array}\right)=:\left(\begin{array}{l}
v_{1} \\
v_{2}
\end{array}\right)
$$

Algebraic calculations give

$$
Q_{\epsilon}^{(2)}(t) A(t)-A^{*}(t) Q_{\epsilon}^{(2)}(t)=2 \epsilon^{2}\left(\begin{array}{cc}
0 & 1 \\
-1 & 0
\end{array}\right)
$$

therefore

$$
\begin{aligned}
& i\left(\left(Q_{\epsilon}^{(2)}(t) A(t)-A^{*}(t) Q_{\epsilon}^{(2)}(t)\right) V(t), V(t)\right) \leq 2 \epsilon^{2} \int 2\left|\operatorname{Im}\left(v_{2} \overline{v_{1}}\right)\right| d t \leq 2 \epsilon \int 2\left|\epsilon v_{1}\right|\left|v_{2}\right| d t \leq \\
& \leq 2 \epsilon \int\left(\epsilon^{2}\left|v_{1}\right|^{2}+\left|v_{2}\right|^{2}\right) d t \leq 2 \epsilon \int\left(\epsilon^{2}+a(t)\right)\left|v_{1}\right|^{2}+\left|v_{2}\right|^{2} d t=\epsilon\left(Q_{\epsilon}^{(2)}(t) V(t), V(t)\right) .
\end{aligned}
$$

Using this estimate in (3.19), we get

$$
\begin{aligned}
& \frac{d}{d t} E_{\epsilon}(t)=\left(\frac{d}{d t} Q_{\epsilon}^{(2)}(t) V(t), V(t)\right)+i \beta\left(\left(Q_{\epsilon}^{(2)} A(t)-A^{*}(t) Q_{\epsilon}^{(2)}\right) V(t), V(t)\right) \leq \\
& \leq\left(\frac{d}{d t} Q_{\epsilon}^{(2)}(t) V(t), V(t)\right)+\beta \epsilon E_{\epsilon}(t) \\
& =\left[\frac{\left(\frac{d}{d t} Q_{\epsilon}^{(2)}(t) V(t), V(t)\right)}{\left(Q_{\epsilon}^{(2)}(t) V(t), V(t)\right)}+\beta \epsilon\right] E_{\epsilon}(t) .
\end{aligned}
$$


In order to apply the Gronwall lemma, we first estimate the integral

$$
\int_{0}^{T} \frac{\left(\frac{d}{d t} Q_{\epsilon}^{(2)}(t) V(t), V(t)\right)}{\left(Q_{\epsilon}^{(2)}(t) V(t), V(t)\right)} d t .
$$

Let us recall that from the definition of the quasi-symmetriser, it follows that

$$
\left(Q_{\epsilon}^{(2)} V, V\right)=2 \int\left(\left(a(t)+\epsilon^{2}\right) \beta^{2}|v|^{2}+\left|\partial_{t} v\right|^{2}\right) d t
$$

Thus, setting $c_{1}:=\max \left(1,2\left(\|a\|_{L^{\infty}}+\epsilon^{2}\right)\right)$, we obtain a bound from above for (3.22), that is

$$
\left(Q_{\epsilon}^{(2)} V, V\right) \leq c_{1}|V|^{2} .
$$

Observing that $\epsilon^{2} c_{1}^{-1} \leq 1$ and $\epsilon^{2} c_{1}^{-1} \leq c_{1}$ for small enough $\epsilon$, we can also deduce an inequality from below of the form

$$
\epsilon^{2} c_{1}^{-1}|V|^{2} \leq\left(Q_{\epsilon}^{(2)} V, V\right)
$$

Hence, there exists a constant $c_{1} \geq 1$ such that for $t \in[0, T]$ we have

$$
c_{1}^{-1} \epsilon^{2}|V(t)|^{2} \leq\left(Q_{\epsilon}^{(2)}(t) V(t), V(t)\right) \leq c_{1}|V(t)|^{2} .
$$

The lower bound, together with [26, Lemma 2] (see [33, Lemma 2] for a detailed proof), allows us to estimate the integral (3.21) as follows

$$
\begin{aligned}
& \int_{0}^{T} \frac{\left(\frac{d}{d t} Q_{\epsilon}^{(2)}(t) V(t), V(t)\right)}{\left(Q_{\epsilon}^{(2)}(t) V(t), V(t)\right)} d t \leq \int_{0}^{T} \frac{\left(\frac{d}{d t} Q_{\epsilon}^{(2)}(t) V(t), V(t)\right)}{\left(Q_{\epsilon}^{(2)}(t) V(t), V(t)\right)^{1-\frac{1}{T}}\left(Q_{\epsilon}^{(2)}(t) V(t), V(t)\right)^{\frac{1}{T}}} d t \leq \\
& \leq c_{1}^{\frac{1}{l}} \epsilon^{-\frac{2}{T}} \int_{0}^{T} \frac{\left(\frac{d}{d t} Q_{\epsilon}^{(2)}(t) V(t), V(t)\right)}{\left(Q_{\epsilon}^{(2)}(t) V(t), V(t)\right)^{1-\frac{1}{l}}|V(t)|^{\frac{2}{T}}} d t \leq c_{1}^{\frac{1}{l}} \epsilon^{-\frac{2}{T}} c_{T}\left\|Q_{\epsilon}^{(2)}\right\|_{\mathcal{C}^{l}([0, T])}^{\frac{1}{l}} \leq c_{3} \epsilon^{-\frac{2}{T}} .
\end{aligned}
$$

Thus, by the Gronwall lemma and the estimates for the quasi-symmetriser just derived, we obtain

$$
E_{\epsilon}(t) \leq E_{\epsilon}(0) e^{c_{3} \epsilon^{-\frac{2}{T}}+\beta \epsilon T} .
$$

Combining the latter inequality with (3.23) we obtain

$$
c_{1}^{-1} \epsilon^{2}|V(t)|^{2} \leq E_{\epsilon}(t) \leq E_{\epsilon}(0) e^{c_{T}\left(\epsilon^{-\frac{2}{l}}+\beta \epsilon\right)} \leq c_{1}|V(0)|^{2} e^{c_{T}\left(\epsilon^{-\frac{2}{T}}+\beta \epsilon\right)} .
$$

We choose $\epsilon$ such that $\epsilon^{-\frac{2}{T}}=\beta \epsilon$, thus $\epsilon=\beta^{-\frac{l}{2+l}}$ and $\epsilon \beta=\beta^{\frac{2}{2+l}}$. We can assume $\beta$ is large enough with a remark for small $\beta$ similar to Case 2 . Setting $\sigma=1+\frac{l}{2}$, for a suitable constant $K \in \mathbb{C}$ it follows that

$$
|V(t)|^{2} \leq C \beta^{\frac{l}{\sigma}} e^{K \beta^{\frac{1}{\sigma}}}|V(0)|^{2}
$$

This means that

$$
\beta^{2}|v(t)|^{2}+\left|v^{\prime}(t)\right|^{2} \leq C \beta^{\frac{l}{\sigma}} e^{K \beta^{\frac{1}{\sigma}}}\left(\beta^{2}\left|v_{0}\right|^{2}+\left|v_{1}\right|^{2}\right)
$$

as required. 


\subsection{Case 4: $a \in \mathcal{C}^{\alpha}([0, T])$, with $0<\alpha<2, a(t) \geq 0$}

In this last case we extend the proof of Case 2. However, under these assumptions the roots of the matrix $A(t)$, that are, $\pm \sqrt{a(t)}$ might coincide, and hence they are Hölder of order $\frac{\alpha}{2}$ instead of $\alpha$. We can refer for the background discussions of this to [25]. In order to adapt this proof to the one for Case 2 we will assume without loss of generality that $a \in \mathcal{C}^{2 \alpha}([0, T])$ with $0<\alpha<1$, so that $\sqrt{a} \in \mathcal{C}^{\alpha}([0, T])$. At the end, to formulate the final result, we will change $\alpha$ into $\frac{\alpha}{2}$.

Following the argument developed for Case 2, we look again for solutions of the form

$$
V(t)=e^{-\rho(t) \beta^{\frac{1}{s}}}(\operatorname{det} H(t))^{-1} H(t) W(t),
$$

with the real-valued function $\rho(t)$, the exponent $s$ and the energy $W(t)$ to be chosen later, while $H(t)$ is the matrix given by

$$
H(t)=\left(\begin{array}{cc}
1 & 1 \\
\lambda_{1, \alpha}^{\epsilon}(t) & \lambda_{2, \alpha}^{\epsilon}(t)
\end{array}\right)
$$

where the regularised eigenvalues of $A(t), \lambda_{1, \alpha}^{\epsilon}(t)$ and $\lambda_{2, \alpha}^{\epsilon}(t)$ differ from the ones defined in the previous case in the following way

$$
\begin{aligned}
& \lambda_{1, \alpha}^{\epsilon}(t):=\left(-\sqrt{a} * \varphi_{\epsilon}\right)(t)+\epsilon^{\alpha}, \\
& \lambda_{2, \alpha}^{\epsilon}(t):=\left(+\sqrt{a} * \varphi_{\epsilon}\right)(t)+2 \epsilon^{\alpha} .
\end{aligned}
$$

Arguing as in Case 2, we can easily see that the smooth functions $\lambda_{1}^{\epsilon}(t)$ and $\lambda_{2}^{\epsilon}(t)$ satisfy, uniformly in $t$ and $\epsilon$ (and trivially in $x$ ), the following inequalities

- $\operatorname{det} H(t)=\lambda_{2, \alpha}^{\epsilon}(t)-\lambda_{1, \alpha}^{\epsilon}(t) \geq c_{1} \epsilon^{\alpha}$;

- $\left|\lambda_{1, \alpha}^{\epsilon}(t)+\sqrt{a(t)}\right| \leq c_{2} \epsilon^{\alpha}$;

- $\left|\lambda_{2, \alpha}^{\epsilon}(t)-\sqrt{a(t)}\right| \leq c_{3} \epsilon^{\alpha}$.

We now look for the energy estimates. In order to do this, recalling the calculations done before (3.13) and (3.14), we obtain

$$
\begin{aligned}
& \frac{d}{d t}|W(t)|^{2}=2 \operatorname{Re}\left(W_{t}(t), W(t)\right) \leq\left(2 \rho^{\prime}(t) \beta^{\frac{1}{s}}+2\left\|H^{-1}(t) H_{t}(t)\right\|+\right. \\
& \left.+2\left|(\operatorname{det} H(t))^{-1} \operatorname{det} H_{t}(t)\right|+\beta\left\|H^{-1} A H-\left(H^{-1} A H\right)^{*}\right\|\right)|W(t)|^{2} .
\end{aligned}
$$

The same arguments as in Case 2 allow us to get the following bounds

I) $\left\|H^{-1}(t) H_{t}(t)\right\| \leq k_{1} \epsilon^{-1}$;

II) $\left|(\operatorname{det} H(t))^{-1} \operatorname{det} H_{t}(t)\right| \leq k_{2} \epsilon^{-1}$;

III) $\left\|H^{-1} A H-\left(H^{-1} A H\right)^{*}\right\| \leq k_{3} \epsilon^{\alpha}$.

Combining (3.24) with I), II) and III) we obtain

$$
\frac{d}{d t}|W(t)|^{2} \leq\left(2 \rho^{\prime}(t) \beta^{\frac{1}{s}}+2 k_{1} \epsilon^{-1}+2 k_{2} \epsilon^{-1}+k_{3} \beta \epsilon^{\alpha}\right)|W(t)|^{2} .
$$


We choose $\epsilon^{-1}=\beta \epsilon^{\alpha}$ which yields $\epsilon=\beta^{-\frac{1}{\alpha+1}}$. Thus, setting $\gamma:=\frac{1}{\alpha+1}$, we obtain for a constant $c>0$ the estimate

$$
\frac{d}{d t}|W(t)|^{2} \leq\left(2 \rho^{\prime}(t) \beta^{\frac{1}{s}}+2 c \beta^{\gamma}\right)|W(t)|^{2} .
$$

We take $\rho(t):=\rho(0)-K t$ with $K>0$ to be chosen later. Considering

$$
\frac{1}{s}>\gamma \quad \Longleftrightarrow \quad s<1+\alpha,
$$

we get

$$
\frac{d}{d t}|W(t)|^{2} \leq\left(-2 K+2 c \beta^{\gamma-\frac{1}{s}}\right) \beta^{\frac{1}{s}}|W(t)|^{2} \leq 0,
$$

provided that $\beta$ is large enough. Similarly to Case 2 , we then get

$$
|V(t)| \leq e^{K t \beta^{\frac{1}{s}}}(\operatorname{det} H(t))^{-1} \operatorname{det} H(0)\|H(t)\|(\|H(0)\|)^{-1}|V(0)| .
$$

Since

$$
(\operatorname{det} H(t))^{-1} \operatorname{det} H(0)\|H(t)\|(\|H(0)\|)^{-1} \leq c \epsilon^{-\alpha}=c \beta^{\frac{\alpha}{\alpha+1}},
$$

the inequality (3.25) becomes

$$
|V(t)| \leq c \beta^{\frac{\alpha}{\alpha+1}} e^{K t \beta^{\frac{1}{s}}}|V(0)|
$$

which means

$$
\beta^{2}|v(t)|^{2}+\left|v^{\prime}(t)\right|^{2} \leq c \beta^{\frac{\alpha}{\alpha+1}} e^{K t \beta^{\frac{1}{s}}}\left(\beta^{2}\left|v_{0}\right|^{2}+\left|v_{1}\right|^{2}\right) .
$$

Combining this with a remark for small $\beta$ similar to Case 2 yields the result. Thus Proposition 3.1 is proved.

\section{Proof of Theorem 1.1}

In this section we combine the things from Sect. 2 and Sect. 3 to prove Theorem 1.1. However, we will need one more ingredient, the Fourier transform on $G$, that we now briefly describe.

Let $f \in L^{1}(G)$ and let $\pi \in \widehat{G}$. By a usual abuse of notation we will identify irreducible unitary representations with their equivalence classes. The group Fourier transform of $f$ at $\pi$ is defined by

$$
\mathcal{F}_{G} f(\pi) \equiv \widehat{f}(\pi) \equiv \pi(f):=\int_{G} f(x) \pi(x)^{*} d x,
$$

with integration against the biinvariant Haar measure on $G$. This gives a linear mapping $\widehat{f}(\pi): \mathcal{H}_{\pi} \rightarrow \mathcal{H}_{\pi}$ that can be represented by an infinite matrix once we choose a basis for the Hilbert space $\mathcal{H}_{\pi}$. Consequently, we can write

$$
\mathcal{F}_{G}(\mathcal{R} f)(\pi)=\pi(\mathcal{R}) \widehat{f}(\pi) .
$$


By Kirillov's orbit method (see e.g. [14]), one can explicitly construct the Plancherel measure $\mu$ on the dual $\widehat{G}$. Therefore we can have the Fourier inversion formula. In addition, the operator $\pi(f)=\widehat{f}(\pi)$ is Hilbert-Schmidt:

$$
\|\pi(f)\|_{\mathrm{HS}}^{2}=\operatorname{Tr}\left(\pi(f) \pi(f)^{*}\right)<\infty,
$$

and the function $\widehat{G} \ni \pi \mapsto\|\pi(f)\|_{\mathrm{HS}}^{2}$ in integrable with respect to $\mu$. Furthermore, the Plancherel formula holds:

$$
\int_{G}|f(x)|^{2} d x=\int_{\widehat{G}}\|\pi(f)\|_{\mathrm{HS}}^{2} d \mu(\pi),
$$

see e.g. [14] or [18].

Proof of Theorem 1.1 Our aim is to reduce the Cauchy problem (1.1) to a form allowing us to apply Proposition 3.1. In order to do this, we take the group Fourier transform of (1.1) with respect to $x \in G$ for all $\pi \in \widehat{G}$, that is,

$$
\partial_{t}^{2} \widehat{u}(t, \pi)+a(t) \pi(\mathcal{R}) \widehat{u}(t, \pi)=0 .
$$

Keeping in mind the form (2.4) of the infinitesimal representation $\pi(\mathcal{R})$ Eq. (4.2) can be seen componentwise as an infinite system of equations of the form

$$
\partial_{t}^{2} \widehat{u}(t, \pi)_{m, k}+a(t) \pi_{m}^{2} \widehat{u}(t, \pi)_{m, k}=0,
$$

where we are considering any $\pi \in \widehat{G}$, and any $m, k \in \mathbb{N}$. The key point of the following argument is to decouple the system given by the matrix equation (4.2). In order to do this, we fix an arbitrary representation $\pi$, and a general entry $(m, k)$ and we treat each equation given by (4.3) individually. Note that eventually $\widehat{u}(t, \pi)_{m, k}$ is a function only of $t$. Formally, recalling the notation used in Proposition 3.1, we write

$$
v(t):=\widehat{u}(t, \pi)_{m, k}, \quad \beta^{2}:=\pi_{m}^{2},
$$

and

$$
v_{0}:=\widehat{u}_{0}(\pi)_{m, k}, \quad v_{1}:=\widehat{u}_{1}(\pi)_{m, k} .
$$

Therefore, equation (4.3) becomes

$$
v^{\prime \prime}(t)+a(t) \beta^{2} v(t)=0 .
$$

We proceed discussing implications of Proposition 3.1 separately in each case.

Case 1: $\boldsymbol{a} \in \operatorname{Lip}([0, T]), \boldsymbol{a}(\boldsymbol{t}) \geq \boldsymbol{a}_{\mathbf{0}}>\mathbf{0} \quad$ Applying Proposition 3.1, we get that there exists a positive constant $C>0$ such that

$$
\beta^{2}|v(t)|^{2}+\left|v^{\prime}(t)\right|^{2} \leq C\left(\beta^{2}\left|v_{0}\right|^{2}+\left|v_{1}\right|^{2}\right),
$$

which is equivalent to

$$
\left|\pi_{m} \widehat{u}(t, \pi)_{m, k}\right|^{2}+\left|\widehat{u}^{\prime}(t, \pi)_{m, k}\right|^{2} \leq C\left(\left|\pi_{m} \widehat{u}_{0}(\pi)_{m, k}\right|^{2}+\left|\widehat{u}_{1}(\pi)_{m, k}\right|^{2}\right) .
$$


This holds uniformly in $\pi \in \widehat{G}$ and $m, k \in \mathbb{N}$. We multiply the inequality (4.4) by $\pi_{m}^{4 s / v}$ yielding

$$
\left|\pi_{m}^{1+\frac{2 s}{v}} \widehat{u}(t, \pi)_{m, k}\right|^{2}+\left|\pi_{m}^{\frac{2 s}{v}} \widehat{u}^{\prime}(t, \pi)_{m, k}\right|^{2} \leq C\left(\left|\pi_{m}^{1+\frac{2 s}{v}} \widehat{u}_{0}(\pi)_{m, k}\right|^{2}+\left|\pi_{m}^{\frac{2 s}{v}} \widehat{u}_{1}(\pi)_{m, k}\right|^{2}\right) .
$$

Thus, recalling that for any Hilbert-Schmidt operator $A$ we have

$$
\|A\|_{\mathrm{HS}}^{2}=\sum_{m, k}\left|\left(A \varphi_{m}, \varphi_{k}\right)\right|^{2}
$$

for any orthonormal basis $\left\{\varphi_{1}, \varphi_{2}, \ldots\right\}$, we can consider the infinite sum over $m, k$ of the inequalities provided by (4.5), to get

$$
\begin{aligned}
\left\|\pi(\mathcal{R})^{\frac{1}{2}+\frac{s}{v}} \widehat{u}(t, \pi)\right\|_{\mathrm{HS}}^{2}+\left\|\pi(\mathcal{R})^{\frac{s}{v}} \partial_{t} \widehat{u}(t, \pi)\right\|_{\mathrm{HS}}^{2} \leq \\
\quad \leq C\left(\left\|\pi(\mathcal{R})^{\frac{1}{2}+\frac{s}{v}} \widehat{u}_{0}(\pi)\right\|_{\mathrm{HS}}^{2}+\left\|\pi(\mathcal{R})^{\frac{s}{v}} \widehat{u}_{1}(\pi)\right\|_{\mathrm{HS}}^{2}\right) .
\end{aligned}
$$

We can now integrate both sides of (4.6) against the Plancherel measure $\mu$ on $\widehat{G}$, so that the Plancherel identity yields estimate (1.9).

Case 2: $\boldsymbol{a} \in \mathcal{C}^{\alpha}([\mathbf{0}, \boldsymbol{T}])$, with $\mathbf{0}<\boldsymbol{\alpha}<\mathbf{1}, \boldsymbol{a}(\boldsymbol{t}) \geq \boldsymbol{a}_{\mathbf{0}}>\mathbf{0} \quad$ The application of Proposition 3.1 implies the existence of two positive constants $C, K>0$ such that for all $m, k \in \mathbb{N}$ and for every representation $\pi \in \widehat{G}$ we have

$$
\left|\pi_{m} \widehat{u}(t, \pi)_{m, k}\right|^{2}+\left|\widehat{u}^{\prime}(t, \pi)_{m, k}\right|^{2} \leq C e^{K t \pi_{m}^{\frac{1}{s}}}\left(\left|\pi_{m} \widehat{u}_{0}(\pi)_{m, k}\right|^{2}+\left|\widehat{u}_{1}(\pi)_{m, k}\right|^{2}\right),
$$

where

$$
s<1+\frac{\alpha}{1-\alpha} .
$$

If the Cauchy data $\left(u_{0}, u_{1}\right)$ are in $\gamma_{\mathcal{R}}^{s}(G) \times \gamma_{\mathcal{R}}^{s}(G)$ then there exist two positive constants $A_{0}$ and $A_{1}$ such that

$$
\left\|e^{A_{0} \mathcal{R} \frac{1}{2 s}} u_{0}\right\|_{L^{2}}<\infty \quad \text { and }\left\|e^{A_{1} \mathcal{R}^{\frac{1}{2 s}}} u_{1}\right\|_{L^{2}}<\infty .
$$

We note that we can restrict to consider $\pi_{m}$ large enough since the cut-off to bounded $\pi_{m}$ produces functions in any Gevrey spaces. Indeed, if a cut-off $\chi: \mathbb{R} \rightarrow \mathbb{C}$ has a compact support, then by the same energy estimate, since $\chi(\mathcal{R})$ and $\mathcal{R}$ commute, the problem is reduced to the solution $\chi(\mathcal{R}) u(t, x)$ to the Cauchy problem

$$
\begin{cases}\partial_{t}^{2}(\chi(\mathcal{R}) u(t, x))+a(t) \mathcal{R}(\chi(\mathcal{R}) u(t, x))=0, & (t, x) \in[0, T] \times G, \\ \chi(\mathcal{R}) u(0, x)=\chi(\mathcal{R}) u_{0}(x), & x \in G, \\ \partial_{t}(\chi(\mathcal{R}) u(0, x))=\chi(\mathcal{R}) u_{1}(x), & x \in G,\end{cases}
$$

with data $\chi(\mathcal{R}) u_{0}$ and $\chi(\mathcal{R}) u_{1}$, so it is in any Gevrey class.

Take now $A=\min \left\{A_{0}, A_{1}\right\}$, then we can always assume $K$ in Case 2 of Proposition 3.1 is small enough, so that we have some $B>0$ such that $K T=A-B$. Therefore, we can rewrite inequality (4.7) as

$$
e^{B \pi_{m}^{\frac{1}{s}}}\left(\left|\pi_{m} \widehat{u}(t, \pi)_{m, k}\right|^{2}+\left|\widehat{u}^{\prime}(t, \pi)_{m, k}\right|^{2}\right) \leq C e^{A \pi_{m}^{\frac{1}{s}}}\left(\left|\pi_{m} \widehat{u}_{0}(t)_{m, k}\right|^{2}+\left|\widehat{u}_{1}(t)_{m, k}\right|^{2}\right) .
$$


Summing over $m, k$, integrating against the Plancherel measure of $\widehat{G}$ and applying the Plancherel identity, inequality (4.9) becomes

$$
\begin{aligned}
\left\|e^{B \mathcal{R} \frac{1}{2 s}} u\right\|_{L^{2}(G)} & +\left\|e^{B \mathcal{R} \frac{1}{2 s}} \partial_{t} u\right\|_{L^{2}(G)} \leq\left\|e^{B \mathcal{R} \frac{1}{2 s}} \mathcal{R}^{\frac{1}{2}} u\right\|_{L^{2}(G)}+\left\|e^{B \mathcal{R} \frac{1}{2 s}} \partial_{t} u\right\|_{L^{2}(G)} \leq \\
& \leq\left\|e^{A \mathcal{R} \frac{1}{2 s}} \mathcal{R}^{\frac{1}{2}} u_{0}\right\|_{L^{2}(G)}+\left\|e^{A \mathcal{R}} \frac{1}{2 s} u_{1}\right\|_{L^{2}(G)} .
\end{aligned}
$$

If a function $f$ belongs to $\gamma_{\mathcal{R}}^{s}(G)$, then also $\mathcal{R}^{\frac{1}{2}} f$ is in $\gamma_{\mathcal{R}}^{s}(G)$. Therefore, from (4.10) we get the desired well-posedness result.

Case 3: $a \in \mathcal{C}^{l}([0, T])$, with $l \geq 2, a(t) \geq 0$ Similarly to the previous cases, the application of Proposition 3.1 yields the existence of two positive constants $C, K>0$ such that

$$
\begin{aligned}
\left|\pi_{m} \widehat{u}(t, \pi)_{m, k}\right|^{2}+\left|\widehat{u}^{\prime}(t, \pi)_{m, k}\right|^{2} & \leq C \pi_{m}^{\frac{l}{\sigma}+2} e^{K T \pi_{m}^{\frac{1}{\sigma}}}\left|\widehat{u}_{0}(\pi)_{m, k}\right|^{2}+C \pi_{m}^{\frac{l}{\sigma}} e^{K T \pi_{m}^{\frac{1}{\sigma}}}\left|\widehat{u}_{1}(\pi)_{m, k}\right|^{2} \leq \\
& \leq C e^{K^{\prime} \pi_{m}^{\frac{1}{s}}}\left|\widehat{u}_{0}(\pi)_{m, k}\right|^{2}+C e^{K^{\prime} \pi_{m}^{\frac{1}{s}}}\left|\widehat{u}_{1}(\pi)_{m, k}\right|^{2},
\end{aligned}
$$

with $1 \leq s<\sigma=1+\frac{l}{2}$, for some $K^{\prime}>0$ small enough. Proceeding as in Case 2, we obtain the desired inequality, and hence also the well-posedness result.

Case 4: $a \in \mathcal{C}^{\alpha}([0, T])$, with $0<\alpha<2, a(t) \geq 0$ In this last case, applying Proposition 3.1 we have that there exist two positive constants $C, K>0$ such that

$$
\pi_{m}^{2}\left|\widehat{u}(t, \pi)_{m, k}\right|^{2}+\left|\widehat{u}^{\prime}(t, \pi)_{m, k}\right|^{2} \leq C \pi_{m}^{\frac{\alpha / 2}{\alpha / 2+1}} e^{K T \pi_{m}^{\frac{1}{S}}}\left(\pi_{m}^{2}\left|\widehat{u}_{0}(\pi)_{m, k}\right|^{2}+\left|\widehat{u}_{1}(\pi)_{m, k}\right|^{2}\right),
$$

with $1 \leq s<1+\frac{\alpha}{2}$. Arguing as above, the result follows.

Publisher's Note Springer Nature remains neutral with regard to jurisdictional claims in published maps and institutional affiliations.

Open Access This article is licensed under a Creative Commons Attribution 4.0 International License, which permits use, sharing, adaptation, distribution and reproduction in any medium or format, as long as you give appropriate credit to the original author(s) and the source, provide a link to the Creative Commons licence, and indicate if changes were made. The images or other third party material in this article are included in the article's Creative Commons licence, unless indicated otherwise in a credit line to the material. If material is not included in the article's Creative Commons licence and your intended use is not permitted by statutory regulation or exceeds the permitted use, you will need to obtain permission directly from the copyright holder. To view a copy of this licence, visit http://creativecommons.org/licenses/by/4.0/.

\section{References}

1. Beals, R.: Opérateurs invariants hypoelliptiques sur un groupe de Lie nilpotent. In: Séminaire GoulaouicSchwartz 1976/1977: Équations aux dérivées partielles et analyse fonctionnelle (1977). Exp. No. 19:8 pp.

2. Bony, J.-M., Schapira, P.: Existence et prolongement des solutions holomorphes des équations aux dérivées partielles. Invent. Math. 17, 95-105 (1972)

3. Bronšteı̌n, M.D.: The Cauchy problem for hyperbolic operators with characteristics of variable multiplicity. Tr. Mosk. Mat. Obŝ. 41, 83-99 (1980)

4. Cicognani, M., Colombini, F.: The Cauchy problem for p-evolution equations. Trans. Am. Math. Soc. 362, 4853-4869 (2010) 
5. Cicognani, M., Hirosawa, F., Reissig, M.: The log-effect for p-evolution type models. J. Math. Soc. Jpn. 60, 819-863 (2008)

6. Cicognani, M., Hirosawa, F., Reissig, M.: Loss of regularity for p-evolution type models. J. Math. Anal. Appl. 347, 35-58 (2008)

7. Colombini, F., Kinoshita, T.: On the Gevrey well-posedness of the Cauchy problem for weakly hyperbolic equations of higher order. J. Differ. Equ. 186, 394-419 (2002)

8. Colombini, F., Lerner, N.: Hyperbolic operators with non-Lipschitz coefficients. Duke Math. J. 77, 657698 (1995)

9. Colombini, F., Métivier, G.: The Cauchy problem for wave equations with non Lipschitz coefficients; application to continuation of solutions of some nonlinear wave equations. Ann. Sci. Éc. Norm. Supér. 4(41), 177-220 (2008)

10. Colombini, F., Métivier, G.: Counterexamples to the well posedness of the Cauchy problem for hyperbolic systems. Anal. PDE 8, 499-511 (2015)

11. Colombini, F., Spagnolo, S.: An example of a weakly hyperbolic Cauchy problem not well posed in $\mathcal{C}^{\infty}$. Acta Math. 148, 243-253 (1982)

12. Colombini, F., De Giorgi, E., Spagnolo, S.: Sur les équations hyperboliques avec des coefficients qui ne dépendent que du temps. Ann. Sc. Norm. Super. Pisa, Cl. Sci. 6, 511-559 (1979)

13. Colombini, F., Jannelli, E., Spagnolo, S.: Nonuniqueness in hyperbolic Cauchy problems. Ann. Math. (2) 126(3), 495-524 (1987)

14. Corwin, L.J., Greenleaf, F.P.: Representations of Nilpotent Lie Groups and Their Applications: Basic Theory and Examples. Cambridge Studies in Advanced Mathematics, vol. 18. Cambridge University Press, Cambridge (1990)

15. D'Ancona, P., Spagnolo, S.: Quasi-symmetrization of hyperbolic systems and propagation of the analytic regularity. Boll. Unione Mat. Ital., B 1, 169-186 (1998)

16. Dasgupta, D., Ruzhansky, M.: Gevrey functions and ultradistributions on compact Lie groups and homogeneous spaces. Bull. Sci. Math. 138, 756-782 (2014)

17. Dasgupta, D., Ruzhansky, M.: Eigenfunction expansions of ultradifferentiable functions and ultradistributions. Trans. Am. Math. Soc. 368, 8481-8498 (2016)

18. Fischer, V., Ruzhansky, M.: Quantization on Nilpotent Lie Groups. Progress in Mathematics, vol. 314. Birkhäuser, Basel (2016) (open access book)

19. Fischer, V., Ruzhansky, M.: Sobolev spaces on graded groups. Ann. Inst. Fourier 67, 1671-1723 (2017)

20. Fischer, V., Ruzhansky, M., Taranto, C.: On the sub-Laplacian Gevrey spaces. Math. Nachr. (2020). https://doi.org/10.1002/mana.202000295. arXiv:1805.08667v3

21. Folland, G.B.: Subelliptic estimates and function spaces on nilpotent Lie groups. Ark. Mat. 13(2), 161207 (1975)

22. Folland, G.B.: Harmonic Analysis in Phase Space, 2nd edn. Princeton University Press, Princeton (1989)

23. Folland, G.B., Stein, E.M.: Estimates for the $\overline{\partial_{b}}$ complex and analysis on the Heisenberg group. Commun. Pure Appl. Math. 27, 429-522 (1974)

24. Folland, G.B., Stein, E.M.: Hardy Spaces on Homogeneous Groups. Mathematical Notes, vol. 28. Princeton University Press/University of Tokyo Press, Princeton/Tokyo (1982)

25. Garetto, C., Ruzhansky, M.: On the well-posedness of weakly hyperbolic equations with time-dependent coefficients. J. Differ. Equ. 253, 1317-1340 (2012)

26. Garetto, C., Ruzhansky, M.: Weakly hyperbolic equations with non-analytic coefficients and lower order terms. Math. Ann. 357, 401-440 (2013)

27. Garetto, C., Ruzhansky, M.: Wave equation for sum of squares on compact Lie groups. J. Differ. Equ. 258, 4324-4347 (2015)

28. Garetto, C., Ruzhansky, M.: Hyperbolic second order equations with non-regular time dependent coefficients. Arch. Ration. Mech. Anal. 217, 113-154 (2015)

29. Helffer, B., Nourrigat, J.: Caracterisation des opérateurs hypoelliptiques homogènes invariants à gauche sur un groupe de Lie nilpotent gradué. Commun. Partial Differ. Equ. 4(8), 899-958 (1979)

30. Helgason, S.: Wave equations on homogeneous spaces. In: Lie Group Representations, III, College Park, Md., 1982/1983. Lecture Notes in Math., vol. 1077, pp. 254-287. Springer, Berlin (1984)

31. Hörmander, L.: Hypoelliptic second order differential equations. Acta Math. 119, 147-171 (1967)

32. Hulanicki, A., Jenkins, J.W., Ludwig, J.: Minimum eigenvalues for positive, Rockland operators. Proc. Am. Math. Soc. 94, 718-720 (1985)

33. Kinoshita, T., Spagnolo, S.: Hyperbolic equations with non-analytic coefficients. Math. Ann. 336, 551569 (2006)

34. Melrose, R.: Propagation for the wave group of a positive subelliptic second-order differential operator. In: Hyperbolic Equations and Related Topics, Katata/Kyoto, 1984, pp. 181-192. Academic Press, Boston (1986) 
35. Miller, K.G.: Parametrices for hypoelliptic operators on step two nilpotent Lie groups. Commun. Partial Differ. Equ. 5(11), 1153-1184 (1980)

36. Müller, D., Stein, E.M.: $L^{p}$-estimates for the wave equation on the Heisenberg group. Rev. Mat. Iberoam. 15(2), 297-334 (1999)

37. Nachman, A.I.: The wave equation on the Heisenberg group. Commun. Partial Differ. Equ. 7(6), 675-714 (1982)

38. Nishitani, T.: Sur les équations hyperboliques à coefficients höldériens en $t$ et de classe de Gevrey en $x$. Bull. Sci. Math. (2) 107(2), 113-138 (1983)

39. Reed, M., Simon, B.: Methods of Modern Mathematical Physics, Vol. 1: Functional Analysis, revised and enlarged edn. Academic Press, San Diego (1980)

40. Rockland, C.: Hypoellipticity on the Heisenberg group-representation-theoretic criteria. Trans. Am. Math. Soc. 240, 1-52 (1978)

41. Rothschild, L.P., Stein, E.M.: Hypoelliptic differential operators and nilpotent groups. Acta Math. 137(34), 247-320 (1976)

42. Ruzhansky, M., Tokmagambetov, N.: Wave equation for operators with discrete spectrum and irregular propagation speed. Arch. Ration. Mech. Anal. 226, 1161-1207 (2017)

43. Ruzhansky, M., Turunen, V.: Pseudo-Differential Operators and Symmetries: Background Analysis and Advanced Topics. Birkhäuser, Basel (2009)

44. Ruzhansky, M., Yessirkegenov, N.: Very weak solutions to hypoelliptic wave equations. J. Differ. Equ. 268, 2063-2088 (2020)

45. Taylor, M.E.: Noncommutative Harmonic Analysis. Mathematical Surveys and Monographs, vol. 22. Am. Math. Soc., Providence (1986)

46. ter Elst, A.F.M., Robinson, D.W.: Spectral estimates for positive Rockland operators. In: Algebraic Groups and Lie Groups. Austral. Math. Soc. Lect. Ser., vol. 9, pp. 195-213. Cambridge University Press, Cambridge (1997)

47. Thangavelu, S.: Harmonic Analysis on the Heisenberg Group. Birkhäuser, Boston (1998) 